\title{
Comparison of Three Methods of Clinical Diagnosis, Microscopic and PCR Techniques for Detection of Trichomoniasis in Women in the Yasuj City
}

\author{
Moshfe Abdolali ${ }^{1}$, Khozouei Ashkzari Zohreh ${ }^{2}$, Aramesh Shahintaj ${ }^{3}$, Ghaffari Parvin ${ }^{3}$, \\ Ali Jamshidi ${ }^{4}$, * \\ ${ }^{1}$ Cellular and Molecular Research Center, Yasuj University of Medical Sciences, Yasuj, Iran \\ ${ }^{2}$ Student Research Committee, Yasuj University of Medical Sciences, Yasuj, Iran \\ ${ }^{3}$ Gynecologist, Yasuj University of Medical Sciences, Yasuj, Iran \\ ${ }^{4}$ Department of Parasitology and Mycology, Yasuj University of Medical Sciences, Yasuj, Iran \\ Email address: \\ jamshidi@zaums.ac.ir (A. Jamshidi), jamshidiali91@yahoo.com (A. Jamshidi)
}

\section{To cite this article:}

Moshfe Abdolali, Khozouei Ashkzari Zohreh, Aramesh Shahintaj, Ghaffari Parvin, Ali Jamshidi. Comparison of Three Methods of Clinical Diagnosis, Microscopic and PCR Techniques for Detection of Trichomoniasis in Women in the Yasuj City. Science Journal of Clinical Medicine. Vol. 5, No. 1, 2016, pp. 12-15. doi: 10.11648/j.sjcm.20160501.12

\begin{abstract}
Trichomoniasis is a protozoan disease caused by Trichomonas vaginalis and the most common non- viral sexually transmitted disease. It is the third most common agent of vaginitis. Diagnosis of the disease is based on laboratory wet smear test. In this study, we compared three methods (Clinical diagnosis, Microscopic and Polymerase Chain Reaction) who refered to Yasuj University Women's clinic.in this cross- sectional study, 100 women with vaginitis clinical symptoms selected and after clinical diagnosis, their personal information recorded in a separate questionnaire. Vaginal samples prepared for wet smear test and PCR. Their vaginal discharge was studied in 100 patients, 23(23\%) cases of clinical examination, Trichomoniasis infection was diagnosed and also 33(33\%) cases infected Trichomoniasis in combination with other vaginitis. $41(41 \%)$ were positive in direct microscopic observation and 11(11\%) cases were positive by PCR. From 41 positive cases in wet smear diagnosis, 28 cases were positive in clinical diagnosis, from 11 positive cases in PCR method, only 4 cases is positive in wet smear diagnosis and 4 cases is positive in clinical diagnosis. Statistically, was not found significant differences between diagnosis the clinical, microscopic and PCR. Also was not found significant difference between clinical Symptoms and diagnosis of Trichomoniasis, therefore the clinical signs alone cannot be used in the diagnosis of Trichomonas vaginalis and the reliance on clinical diagnosis of Trichomoniasis in more than $60 \%$ positive cases, which are not detected as a result of the treatment are unnecessary. PCR has a sensitivity and specificity of $100 \%$ which can be quickly and accurately identify the correct data and be treated properly. Improved T. vaginalis control efforts are imperative and require better disease recognition, clinical application of sensitive nucleic acid-based tests, and management of sexual partners.
\end{abstract}

Keywords: Trichomoniasis, Clinical Diagnosis, Microscopic Diagnosis, PCR, Yasuj

\section{Introduction}

Trichomoniasis is caused by the protozoan parasite Trichomonas vaginalis. It is one of the most common causes of vaginitis worldwide, with an estimated 170 million cases occurring annually. This disease accounts for $4-35 \%$ of vaginitis diagnosed in symptomatic women presenting in primary care settings. [1] In women, Trichomonas vaginalis infect the lower urogenital tract, causing superficial vaginal and cervical ulceration. It has been associated with adverse pregnancy outcome and atypical pelvic inflammatory disease and has been suggested to have a role in HIV transmission [2]. Typical symptoms include frothy yellow discharge, itch, odour, dyspareunia and occasionally vaginal bleeding. Infection of the urethra and Para urethral glands causes dysuria and frequency [3]. Traditionally, gynecologists rely on the clinical picture of Trichomoniasis; however, this can be misleading because typical clinical signs appear in just $12 \%$ patients with Trichomonas vaginalis infection. It is known that Trichomoniasis can lead to inflammatory small pelvic diseases, reproductive dysfunction and increases the 
risk of premature rupture of fetal membranes and low birth weight. Therefore, in order to accurately diagnose this disease, parasitological investigation is necessary. Different methods are being used for the laboratory diagnosis of trichomoniasis, such as wet mount, various staining methods: Giemsa and acridine orange fluorescent staining, Papanicolaou staining, culture, latex agglutination, enzymelinked immunosorbent assay and more recently, molecular techniques, e.g., polymerase chain reaction (PCR) [4]. Conventional wet mount and culture, such as Trichomonas vaginalis In Pouch commercial system are the most commonly employed methods of Trichomonas vaginalis detection. Giemsa and acridine orange staining methods are seldom encountered in sensitivity and specificity studies engaged in the laboratory diagnosis of trichomoniasis [5-7]. The aim of this study was to compare three methods (Clinical diagnosis, Microscopic and Polymerase Chain Reaction) who refered to Yasuj University Women's clinic. Yasuj is a city in and the capital of Kohgiluye and Boyer-Ahmad Province, Iran. At the 2006 census, its population was 96,786, in 20,297 families. Yasuj is an industrial city in the Zagros Mountains of southwestern Iran. The term "Yasuj" is also used to refer to the entire region.

\section{Materials and Methods}

In this cross- sectional study, 100 women with vaginitis clinical symptoms selected and after clinical diagnosis by obstetricians and completed a clinical questionnaire: A) using the applicator, the posterior fornix of the vaginal secretions were sampled and the sample test tube containing $2 \mathrm{ml}$ of sterile normal saline placed. Then, the samples were transferred to the Department of Parasitological, Yasuj Faculty of Medicine. After half an hour, by forceps, applicator brought out, and put it on the vaginal smear slides stick to the surface. Finally, with the cover glass on the slide, with a magnification of $10 \mathrm{X}$ and $40 \mathrm{X}$, we attempted to observe the microscopic samples (using morphological forms and swirl parasites). B) Swabs were placed in a dry sterile plastic container and were frozen at or below $-20^{\circ} \mathrm{C}$ until they were subjected for PCR (DNA extraction: There are several ways to do this. In this study, DNA was extracted from samples of vaginal, phenol - chloroform was used.400$500 \mu \mathrm{l}$ lysis buffer are added slowly to the case and put 24 hours in water bath at $37^{\circ} \mathrm{C}$. Then add $500 \mu \mathrm{l}$ of phenol and vortex for 5 minutes away and 8,000RPM centrifuges. Double-layer is formed. The upper layer is slowly going on. Throw away the substrate. The upper layer, $500 \mathrm{ml}$ of chloroform is added and give it a shake. Then for 5 minutes with around 8,000 RPM centrifuges and the upper phase, and we have added $3 M$ sodium acetate and about $50 \mu$ l. twice the volume of absolute alcohol is added and shake gently until the DNA strands are seen. 12000 RPM for $10 \mathrm{~min}$ at $4^{\circ} \mathrm{C}$ centrifuge to precipitate the DNA. We remove alcohol and to wash the salts in $100 \mathrm{ml}$ of $70 \%$ alcohol are added to the sediment. After shaking a few times, then 2 minutes with a centrifuge 12000 RPM and alcohol are discharged. After drying micro tubes, 50-100 $\mu$ l distilled water and we added it in the freezer). The PCR was carried out on pooled samples using oligonucleotide primers TVK3 and TVK7. Forward primer-TVK3, 5'AT TGT CGA ACA TTG GTC TTA CCC TC3', reverse primer-TVK7, 5' TCT GTG CCG TCT TCA AGT ATG C3' purified $T$. vaginalis DNA of Shiraz University of Medical Sciences, and sterile water were used as negative controls, respectively [8]. After 1.5\% agarose gel electrophoresis of PCR products using UV light for the presence of DNA bands in the gel duct in the range of proliferation was evaluated. At each stage of PCR testing of DNA Trichomonas vaginalis were used as positive controls. For a negative control sample containing all the PCR, without DNA were used. Information obtained in the clinical diagnosis of vaginitis and the results of the study of microscopic detection of Trichomonas vaginalis, as well as the results of PCR with the variables of interest to the tables of pre-designed shifted and then use the SPSS software and analyzed $\mathrm{K}^{2}$ for results analysis.

Table 1. Volumes ( $\mathrm{ml}$ ) of the constituents of the PCR mix, and amplification procedure used for each of the evaluated PCR and Amplification programmes.

\begin{tabular}{lllllllll}
\hline Primer set & $\begin{array}{l}\text { Total } \\
\text { volume }\end{array}$ & $\mathbf{1 0} \mathbf{~ m M ~ T R I S - H C l ~ ( p H ~ 8 . 3 ) ~}$ & $\mathbf{2 5} \mathbf{~ m M ~ M g C 1 2}$ & $\mathbf{2} \mathbf{~ m M ~ d N T P}$ & $\mathbf{5} \mathbf{~ m M}$ primer & $\begin{array}{l}\text { 0.5 Units } \\
\text { Taq }\end{array}$ & $\begin{array}{l}\text { Milli-Q } \\
\text { water }\end{array}$ & $\begin{array}{l}\text { Sample } \\
\text { extract }\end{array}$ \\
\hline TVK3/7 & 50 & 5 & 5 & 7 & 1.5 & 0.4 & 20.6 & 10 \\
\hline
\end{tabular}

Table 1. Continued.

\begin{tabular}{|c|c|c|c|c|c|c|}
\hline Primer set & Pre-denaturation & Denaturation & Annealing & Extension & Final extension & No of cycles \\
\hline TVK3/7 & $95^{\circ} \mathrm{C}, 5$ minutes & $90^{\circ} \mathrm{C}, 60$ seconds & $60^{\circ} \mathrm{C}, 30$ seconds & $70^{\circ} \mathrm{C}, 120$ seconds & $72^{\circ} \mathrm{C}, 7$ minutes & 35 \\
\hline
\end{tabular}

Table 2. Comparison of three methods of clinical diagnosis, microscopic and $P C R\left(X^{2}=1.49, D F=2, P=0.474\right)$.

\begin{tabular}{|c|c|c|c|c|c|c|c|c|}
\hline \multirow{3}{*}{ PCR } & & & \multicolumn{4}{|c|}{ Microscopic } & \multirow{2}{*}{\multicolumn{2}{|c|}{ PLURAL }} \\
\hline & & & \multicolumn{2}{|c|}{ NEG(-) } & \multicolumn{2}{|c|}{$\operatorname{POS}(+)$} & & \\
\hline & & & $\mathbf{N}$ & $\%$ & $\mathbf{N}$ & $\%$ & $\mathbf{N}$ & $\%$ \\
\hline \multirow{3}{*}{ NEGATIV (-) } & \multirow{3}{*}{$\begin{array}{l}\text { CLINICAL } \\
\text { DIAGNOSIS }\end{array}$} & Candida & 14 & 15.7 & 8 & 9 & 22 & 24.7 \\
\hline & & Vagino Bacterial & 12 & 13.5 & 3 & 3.4 & 13 & 16.8 \\
\hline & & Combined with Trichomonas & 17 & 19.1 & 12 & 13.5 & 29 & 32.6 \\
\hline
\end{tabular}




\begin{tabular}{|c|c|c|c|c|c|c|c|c|}
\hline \multirow{3}{*}{ PCR } & & & \multicolumn{4}{|c|}{ Microscopic } & \multirow{2}{*}{\multicolumn{2}{|c|}{ PLURAL }} \\
\hline & & & \multicolumn{2}{|c|}{ NEG(-) } & \multicolumn{2}{|c|}{ POS $(+)$} & & \\
\hline & & & $\mathbf{N}$ & $\%$ & $\mathbf{N}$ & $\%$ & $\mathbf{N}$ & $\%$ \\
\hline \multirow{4}{*}{ POSITIVE $(+)$} & \multirow{4}{*}{$\begin{array}{l}\text { CLINICAL } \\
\text { DIAGNOSIS }\end{array}$} & Candida & 3 & 27.3 & 2 & 18.2 & 5 & 45.5 \\
\hline & & Vagino Bacterial & 2 & 18.2 & 0 & 0 & 2 & 18.2 \\
\hline & & Combined with Trichomonas & 2 & 18.2 & 2 & 18.2 & 4 & 36.4 \\
\hline & & PLURAL & 7 & 63.6 & 4 & 36.4 & 11 & 100 \\
\hline
\end{tabular}

\section{Results and Discussion}

Study of clinical diagnosis showed $23(23 \%)$ cases with Trichomoniasis and also $33(33 \%)$ cases, Trichomoniasis in combination with other vaginitis. $41(41 \%)$ were positive in direct microscopic observation and $11(11 \%)$ cases were positive by PCR. From 41 positive cases in wet smear diagnosis, 28 cases were positive in clinical diagnosis, from 11 positive cases in PCR method, only 4 cases is positive in microscopic and 4 cases is positive in clinical diagnosis. The results are summarized in Table 3(As indicated in the table below that of the 41 positive samples by microscopy, and 56 cases of clinically diagnosed cases by 11 PCR, only 2 of the three diagnostic methods, has been positive. Between the frequencies of three diagnostic methods, there is no statistically significant correlation $(\mathrm{P}>0.05)$. The clinical diagnosis with any of the diagnostics (microscopy or PCR) there was a statistically significant difference and also distinguish between wet smear and PCR also showed no statistically significant difference. significant difference was not found between clinical signs and diagnosis of Trichomoniasis, therefore the clinical signs alone cannot be used in the diagnosis of Trichomonas vaginalis and the reliance on clinical diagnosis of Trichomoniasis in more than $60 \%$ positive cases, which are not detected as a result of the treatment are unnecessary. PCR has a sensitivity and specificity of $100 \%$ which can be quickly and accurately identify the correct data and be treated properly.

\section{Conclusion}

According to studies and sources, Trichomoniasis diagnosed based on clinical symptoms alone were rejected. Therefore, the emphasis on the use of sensitive and accurate laboratory methods, especially in patients suspected of having chronic diseases seem necessary $[3,9,10]$. In this study, it was determined that the diagnosis of trichomoniasis in women's clinic takes place only on the basis of clinical observations that this method is the only way to diagnose any reference book, is not mentioned. In some cases, vaginitis, non-parasitic pathogen caused by other factors that are difficult to diagnose and treat a drug for treating physician, the patient will be considered. Prepare wet mount in most patients with clinical symptoms, is reliable, but is dependent on the individual and due to the low sensitivity is far from optimal results $[1,2,4,11,12]$. Sensitivity and specificity of clinical diagnosis in comparison to PCR, respectively, 7.1\% and $84 \%$ and $9.7 \%, 88.1 \%$ at the microscopic method was obtained. Therefore, a correct diagnosis can be of additional medication to prevent disease and drug resistance in patients. Unfortunately, based on the evidence available in most parts of the Iran, for the diagnosis of trichomoniasis of any laboratory method is not used, therefore, the problem persists and maternal health as a priority health issues are grappling with a chronic illness and complications The disease also affects them in everyday life and in relationships and what the implications may cause deformities. However, in this study, the objective was to compare three methods for the diagnosis of trichomoniasis was presented. It is hoped that these results and importance of the issue, as in the diagnosis of many diseases, laboratory methods can be used to help in the diagnosis of vaginitis also done this work. Notification and management of sexual partners of women with trichomoniasis are not routinely conducted outside of public clinics, and strategies to enhance these activities are urgently needed. In addition, T. vaginalis screening of at risk men and women should be considered in light of the extent of asymptomatic disease. Substantial evidence has already accrued regarding the high prevalence of trichomoniasis and its associated morbidity, prompting concerns regarding its impact worldwide. Improved T. vaginalis control efforts are imperative and require better disease recognition, clinical application of sensitive nucleic acid-based tests, and management of sexual partners [12-13].

\section{Acknowledgements}

We would like to acknowledge all of the volunteers donating their vaginal samples for this study. This study was supported by a Research Administration Yasuj University of Medical Sciences. (Code No: P/23/14/3/361). Study concept and design: Moshfea Abdolali, Khozouei Ashkzari Zohreh, Aramesh Shahintaj and Ghaffari Parvin. Acquisition of data; Moshfea Abdolali, Khozouei Ashkzari Zohreh, Aramesh Shahintaj, Ghaffari Parvin and Jamshidi Ali. Analysis and interpretation of data: Moshfea Abdolali, Khozouei Ashkzari Zohreh and Jamshidi Ali. Drafting of the manuscript: Jamshidi Ali. Critical revision of the manuscript for important intellectual content: Moshfea Abdolali and Jamshidi Ali. There is no financial disclosure.

\section{References}

[1] P. Simpson, "Real-time PCRs for detection of Trichomonas vaginalis beta-tubulin and $18 \mathrm{~S}$ rRNA genes in female genital specimens", J Med Microbiol, 2007. 56(Pt 6): p. 772-7. 
[2] T. Crucitti, "Comparison of culture and different PCR assays for detection of Trichomonas vaginalis in self collected vaginal swab specimens", Sex Transm Infect, 2003. 79(5): p. 393-8.

[3] M. J. Lusk, "Trichomonas vaginalis: underdiagnosis in urban Australia could facilitate re-emergence", Sex Transm Infect, 2010. 86(3): p. 227-30.

[4] I. V. Radonjic, "Diagnosis of Trichomonas vaginalis infection: The sensitivities and specificities of microscopy, culture and PCR assay", Eur J Obstet Gynecol Reprod Biol, 2006. 126(1): p. 116-20.

[5] J. Knox, "Evaluation of self-collected samples in contrast to practitioner-collected samples for detection of Chlamydia trachomatis, Neisseria gonorrhoeae, and Trichomonas vaginalis by polymerase chain reaction among women living in remote areas", Sex Transm Dis, 2002. 29(11): p. 647-54.

[6] M. Schmitt, "Bead-based multiplex sexually transmitted infection profiling", J Infect, 2014. 69(2): p. 123-33.

[7] B. Van Der Pol, C. S. Kraft, J. A. Williams, "Use of an adaptation of a commercially available PCR assay aimed at diagnosis of chlamydia and gonorrhea to detect Trichomonas vaginalis in urogenital specimens", J Clin Microbiol, 2006. 44(2): p. 366-73.
[8] M. J. Patil, J.M. Nagamoti, S. C. Metgud, "Diagnosis of Trichomonas Vaginalis from Vaginal Specimens by Wet Mount Microscopy, In Pouch TV Culture System, and PCR", J Glob Infect Dis, 2012. 4(1): p. 22-5.

[9] M. M. Hegazi, "Polymerase chain reaction versus conventional methods in the diagnosis of vaginal trichomoniasis", J Egypt Soc Parasitol, 2009. 39(1): p. 11-21.

[10] L. Garcia-Agudo, "Diagnosis of vaginitis-vaginosis by hibridization with DNA strands", Ginecol Obstet Mex, 2013. 81(4): p. 195-200.

[11] J. S Ryu, "Diagnosis of trichomoniasis by polymerase chain reaction", Yonsei Med J, 1999. 40(1): p. 56-60.

[12] A. C. Sena, "Trichomonas vaginalis infection in male sexual partners: implications for diagnosis, treatment, and prevention", Clin Infect Dis, 2007. 44(1): p. 13-22.

[13] S.A. Talari, A. Kazemi, H. Hooshyar, F. Kazemi, M. Arbabi, M.R. Talari, et al "Detection of drug resistance gene in trichomonas vaginalis by PCR", Journal of Kashan University of Medical Sciences, Spring, 2011; Vol. 15, No 1, P. 47-52. 